\title{
Chapter 2 \\ Enhancing Indoor Coverage by \\ Multi-Pairs Copper Cables: The Analog \\ MIMO Radio-over-Copper Architecture
}

\author{
Andrea Matera
}

\begin{abstract}
Nowadays, the majority of indoor coverage issues arise from networks that are mainly designed for outdoor scenarios. Outdoor networks, somewhat uncontrollably, may penetrate indoors with the consequence of coverage holes and outage issues, hence reducing network performances. Moreover, the ever-growing number of devices expected for $5 \mathrm{G}$ worsens this situation, calling for novel bandwidth-efficient, low-latency and cost-effective solutions for indoor wireless coverage. This is the focus of this article, which summarizes the content of my Ph.D. thesis by presenting an analog Centralized Radio Access Network (C-RAN) architecture augmented by copper-cable, possibly pre-existing, to provide dense coverage inside buildings. This fronthaul architecture, referred to as Analog MIMO Radio-over-Copper (AMIMORoC), is an extreme RAN functional-split-option: the all-analog Remote Radio Units take the form of tiny, simple and cheap in-home devices, and Base Band Unit includes also signals' digitization. The A-MIMO-RoC architecture is introduced in this article starting from demonstrating its theoretical feasibility. Then, the origin and evolution of A-MIMO-RoC are described step-by-step by briefly going through previous works based on numerical analysis and simulations results. Finally, the overall discussion is complemented by results obtained with a prototype platform, which experimentally prove the capability of A-MIMO-RoC to extend indoor coverage over the last 100$200 \mathrm{~m}$. Prototype results thus confirm that the proposed A-MIMO-RoC architecture is a valid solution towards the design of dedicated $5 \mathrm{G}$ indoor wireless systems for the billions of buildings which nowadays still suffer from severe indoor coverage issues.
\end{abstract}

This work is a summary of the Ph.D. thesis "Interference Mitigation Techniques in Hybrid WiredWireless Communications Systems for Cloud Radio Access Networks with Analog Fronthauling" [1] supervised by Prof. Umberto Spagnolini.

\footnotetext{
A. Matera (凶)

Dipartimento di Elettronica, Informazione e Bioingegneria (DEIB), Politecnico di Milano, Piazza Leonardo da Vinci, 32, 20133 Milan, Italy

e-mail: andrea.matera@polimi.it

B. Pernici (ed.), Special Topics in Information Technology, PoliMI SpringerBriefs, https://doi.org/10.1007/978-3-030-32094-2_2
} 


\subsection{Introduction}

The goal of this article is to present a novel network architecture, referred to as Analog Multiple-Input Multiple-Output Radio-over-Copper (A-MIMO-RoC), whose goal is to guarantee pervasive indoor wireless coverage within buildings. The role of the proposed A-MIMO-RoC architecture in the fifth generation (5G) of wireless systems is clarified in the following through four main pillar ideas that inspired this work: ( $i$ ) enhance/enable indoor coverage, (ii) Centralized Radio Access Network (C-RAN) architecture, (iii) analog fronthauling and, finally, (iv) the RoC paradigm. These concepts, which will continually recur in the remainder of the paper, are now introduced one-by-one.

\subsubsection{Indoor Propagation and C-RAN Architectures}

By 2020 5G networks will be a reality, and $96 \%$ of wireless data traffic will originate or terminate within a building, with an exponential increase of indoor wireless market value [2]. However, only $2 \%$ of the 30 billion square meters of indoor commercial real estate have dedicated in-building wireless systems, while the remaining are still served by networks originally designed for outdoors. This is the reason why providing enhanced solutions for indoor coverage is the main task that both industries and academia are facing for the deployment of upcoming $5 \mathrm{G}$ systems.

Network deployment densification is almost mandatory to achieve the $5 \mathrm{G}$ performance targets. However, it will cause challenging interference scenarios, thus calling for an actual revolution in the way RAN resources are handled. According to this context, the C-RAN architecture [3], already adopted in 4G networks, will surely play a key role also in the next generation $5 \mathrm{G}$ networks, albeit it will need to be substantially redesigned to accommodate the more demanding $5 \mathrm{G}$ requirements.

\subsubsection{Analog Fronthauling Architectures}

In 4G networks, Remote Radio Units (RRUs) and BaseBand Units (BBUs) communicate over the so-called FrontHaul (FH) link, which is a high-capacity link conventionally based on fiber optics. This architecture is designed to support the streaming of digitized RF signals according to specific digital streaming protocols, such as the Common Public Radio Interface (CPRI) [4].

In perspective, it is widely agreed that today's CPRI-based mobile FH will hardly scale to the increased $5 \mathrm{G}$ radio signal bandwidths, especially for multiple-antenna RRUs. This is mainly due to signal digitization that, beside the uncontrolled endto-end latency, would also cause a severe bandwidth expansion, thus exceeding the capacity of current FH links. In order to overcome these limitations, numerous RAN 


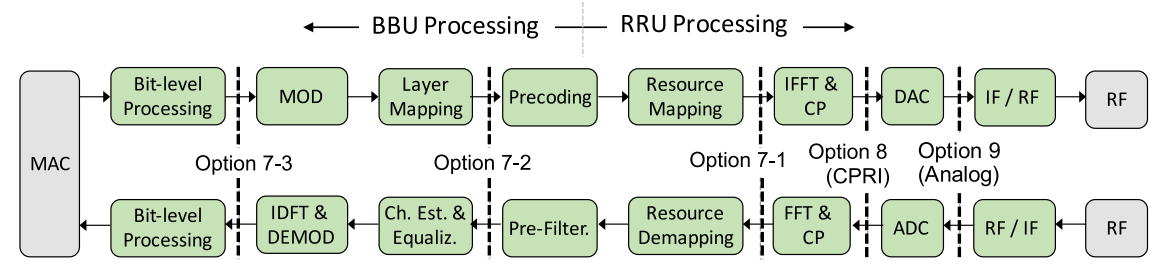

Fig. 2.1 Functional split options proposed for 5G New Radio (NR) and analog fronthaul

architectures have been recently proposed with the aim of making the redistribution of RAN functionalities between BBU and RRU more flexible [5] (see Fig. 2.1).

In contrast with these digital RAN architectures, an effective solution to relax the $\mathrm{FH}$ requirements is the overtaking of conventional digital $\mathrm{FH}$ connections in favor of a fully analog relay of RF signals between RRUs and BBUs. Analog FH links completely by-pass bandwidth/latency issues due to digitization, reduce hardware costs, improve energy efficiency, and easily allow for synchronization among multiple decentralized RRUs, thus enabling any MIMO joint-RRUs processing. As shown in Fig. 2.1, C-RAN architectures with analog FH represent an extreme RAN functional split option whereby even the Analog-to-Digital and Digital-to-Analog Converters (ADC/DAC) are shifted to the BBU, while only the RF functionalities are left to the RRUs, which in turn become extremely low-complexity and analog-based only devices. RRU functionalities are limited to the relaying of Intermediate Frequency (IF) signals to/from the BBUs, after having frequency-converted and scaled down these IF signals in order to comply with FH capabilities. Furthermore, the RRUs used in analog FH are protocol-independent units, hence capable to transparently relay signals belonging to any Radio Access Technology (RAT), which represents an important step towards the wide-range heterogeneity promised by $5 \mathrm{G}$ networks.

\subsubsection{Radio-over-Copper}

Analog C-RAN architectures can be based on different FH technologies. Among these, Analog Radio-over-Fiber (A-RoF), which is based on the analog relaying of optical IF signals over long-range fiber-optic links, is a very attractive $\mathrm{FH}$ architecture capable to carry several Gbps in terms of equivalent wireless data-rate [6]. However, the deployment of a pervasive optical infrastructure would be too costly to provide a satisfactory business case for practical indoor applications. In this case, the most intuitive solution is to extend indoor coverage by relaying the analog $\mathrm{FH}$ signals over the pre-existing Local Area Network (LAN) cabling infrastructure of buildings leading to the so-called A-RoC paradigm [7]. A-RoC architecture allows for the remotization of RF equipment, which are moved in proximity of the User Equipments (UEs) without the need of a new network infrastructure, hence improving indoor 
wireless coverage over the last 100-200 m. Moreover, by leveraging the Power-overEthernet (PoE) technology, A-RoC enables to power the remote RF equipments over the same copper-cables, which simplifies the final architecture as no additional power supply devices are needed. The A-RoC paradigm encompasses both the advantages of analog FH and the economical benefits of reusing the existing copper-cables infrastructures, thus becoming a perfect candidate for extending $5 \mathrm{G}$ indoor wireless coverage as pursued by the telecom industry [8-10].

\subsubsection{Contribution}

This article is intended as a summary of my Ph.D. thesis [1]. Starting from the native A-RoC concept, it presents a more general and flexible analog FH architecture capable to carry multi-RAT/multi-antenna RF signals indoors over 50-200 m coppercables. This novel FH architecture is referred to as Analog-MIMO-RoC, emphasizing the multiple-links nature of both radio (by antennas) and cable (by twisted-pairs) channels connecting the BBU with the UEs.

In particular, this article briefly presents the A-MIMO-RoC architecture from both theoretical and experimental perspectives. In this direction, firstly, the feasibility of the proposed A-MIMO-RoC architecture is demonstrated by numerical results, showing that LAN cables are suitable candidates for relaying RF signals indoors over more than $100 \mathrm{~m}$. Secondly, the evolution of the A-MIMO-RoC architecture is described by reviewing previous works that exhaustively present the theory behind the A-MIMO-RoC architecture. These works cover both uplink and downlink A-MIMORoC channels, single- and multi-UE settings, and also provide useful insights on the performance trade-offs among heterogenous $5 \mathrm{G}$ services when they coexist in the AMIMO-RoC architecture. Lastly, the overall theoretical discussion is complemented by experimental validations of a A-MIMO-RoC prototype for multi-antenna FH indoor applications.

\subsection{The Genesis of the A-MIMO-RoC Architecture}

By reviewing important milestones in the evolution of copper-based FH architectures, this section describes how A-MIMO-RoC origins from the native A-RoC concept.

The A-RoC concept dates back to [7], where twisted-pairs copper-cables were proposed for femto-cell systems to exchange analog RF signals between a remote location hosting all PHY/MAC functionalities (BBU) and an in-home antenna device performing only the analog relay of signals (RRU).

Afterwards, the A-RoC architecture gained lots of attention becoming the basis of commercial solutions exploiting the pre-existing LAN cables of buildings to extend indoor coverage over distances longer than $100 \mathrm{~m}$ [8]. By using all 4 twisted-pairs contained into the LAN cable at low-frequency (characterized by low attenuation 
and crosstalk interference [11]), one can serve up to 4 antennas (e.g., 4 × 4 MIMO) per LAN cable.

Still based on the A-RoC concept, Huang et al. [12] proposed an LTE-over-copper architecture based on the colocation of RRU and Digital Subscriber Line (DSL) equipment in the same street cabinets. Authors proved that, by employing a single twisted-pair in the 21-24 MHz cable frequency band (not used by any DSL service), it is feasible to transport a 3GPP compliant LTE radio signal up to $350 \mathrm{~m}$ away from the cabinet. Crosstalk mitigation in LTE-over-copper systems is covered in Medeiros et al. [13] for the case of 6 twisted-pairs interfering with each other, still in the 21-24 $\mathrm{MHz}$ frequency range.

All the aforementioned works proved the feasibility of A-RoC as an alternative/complementary technology for FH links. However, none of them attempted to push the usage of cable frequency beyond the first tens of $\mathrm{MHz}$, thus not making an efficient usage of the large bandwidth offered by copper-cables. As detailed in the following, this is precisely one of the key characteristics of the A-MIMO-RoC architecture presented here.

\subsection{Theoretical Feasibility and Challenges of A-MIMO-RoC}

This article presents an extension of the A-RoC paradigm to multiple-antennas RRUs and multiple twisted-pairs copper-cables, e.g., LAN cables (i.e., Cat-5/6/7). As shown in Fig. 2.2, the result is a more flexible and general FH architecture, namely

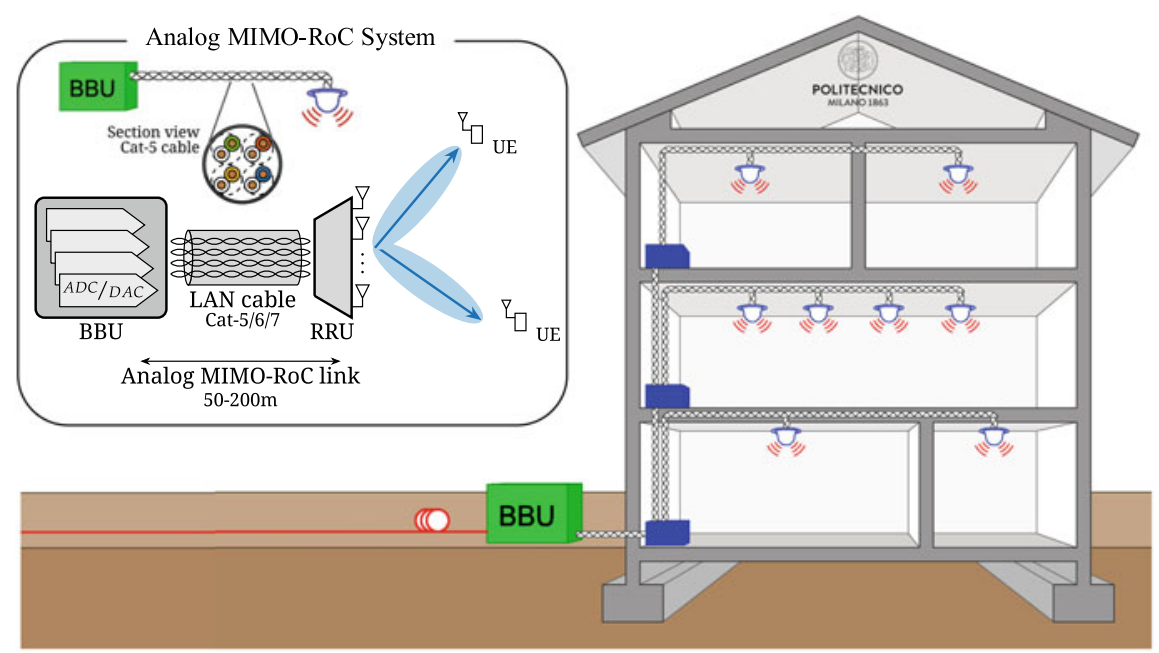

Fig. 2.2 The analog MIMO Radio-over-Copper architecture for indoor wireless coverage 

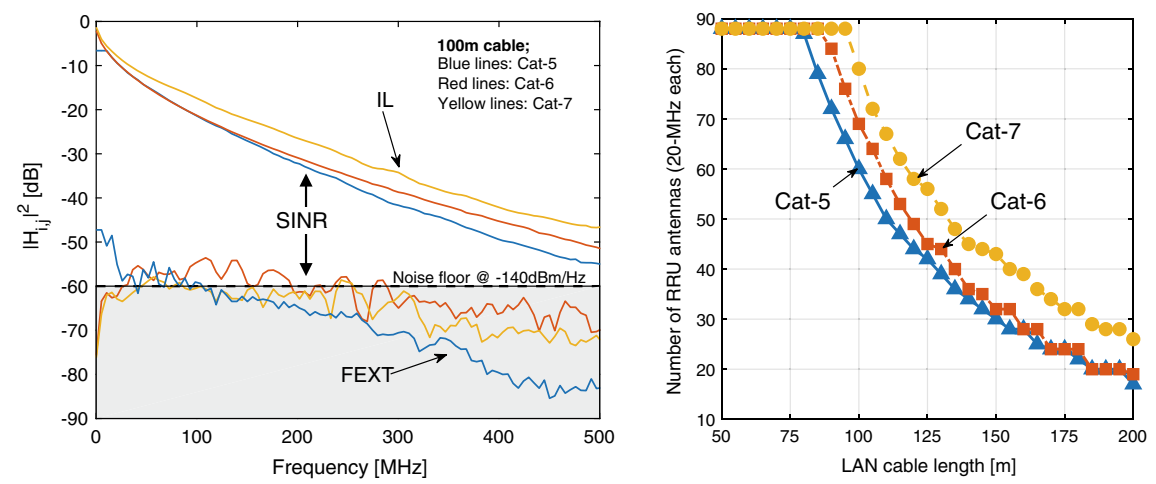

Fig. 2.3 The transport capabilities of LAN cables: IL and FEXT characteristics vs frequency (left) and number of RRU antennas served per LAN cable versus cable type and cable length (right) [11]

A-MIMO-RoC, which is characterized by the cascade of a MIMO wired channel over a MIMO wireless channel. Inspired by the A-RoC concept, an important steppingstone towards the proposal of the A-MIMO-RoC architecture has been to demonstrate the huge transport capabilities offered by LAN cables, which contain 4 twisted-pairs bonded together offering up to $500 \mathrm{MHz}$ bandwidth/pair ( $2 \mathrm{GHz}$ overall). To this aim, the high frequency portion of LAN cables for indoor C-RAN applications has been first explored in our previous work [11] by numerical simulations. As shown in the left part of Fig. 2.3, although cable Insertion Loss (IL) and Far-End-Crosstalk (FEXT) among the 4 pairs rapidly increase versus frequency, the Signal-to-Interference-plusNoise Ratio (SINR) is high enough to allow for transmission up to several hundreds of $\mathrm{MHz}$. This is confirmed by the right portion of Fig. 2.3, which shows that more than 60 RRU antennas carrying a $20 \mathrm{MHz}$ LTE channel/ea. can be served by a $100 \mathrm{~m}$ Cat-5 cable with approx. $500 \mathrm{MHz}$ bandwidth/pair, and this number raises up to approx. 70 and 80 RRU antennas for Cat-6 and Cat-7 LAN cables, respectively.

Previous work [11] asserts the theoretical feasibility of the proposed architecture. However, in practice, the analog nature of A-MIMO-RoC poses several technical challenges that complicate its design and optimization: (i) as mentioned, FEXT among the 4 pairs and IL, if not properly handled, severely limit the performance of LAN cables, especially at high cable frequency (see the left part of Fig. 2.3), (ii) for a large number of RRU antennas, it arises the problem of how to map the signal to/from each antenna onto the available cable resources defined in space dimension (i.e., the 4 twisted-pairs) and frequency dimension (i.e., the frequency bandwidth of each twisted-pair), (iii) the all-analog RRU equipment (e.g., home-device) should be as simple/cheap as possible, but in the meanwhile able to handle up to several tens of antennas, $(i v)$ LAN cables are subject to strict power constraints that must be carefully taken care of in the system design, especially in downlink direction, $(v)$ in case of multiple-UEs, interference cancellation techniques for compound A-MIMO-RoC should be properly designed, but still releasing the RRU from any computationally complex signal processing, and (vi) in heterogeneous 5G networks, the coexistence 
among different services with different constraints in terms of data-rate, reliability and latency must be carefully investigated.

The goal of my Ph.D. research activity has been to propose effective solutions to the problems above. These are briefly presented in the next section.

\subsection{A-MIMO-RoC: Numerical Analysis and Simulations Works}

This section describes the evolution of the A-MIMO-RoC architecture by briefly presenting the main works contributing to build the A-MIMO-RoC theory and addressing the critical issues introduced in the previous section. In this regard, the critical aspects (i), (ii), (iii) mentioned above have been addressed in our previous works $[14,15]$, which present a single-UE uplink A-MIMO-RoC scenario based on LAN cables. In particular, [14] proposes a novel and flexible resource allocation strategy between the RF signals at each RRU antenna, received from the UEs over the wireless channel, and the twisted-pair/frequency resources over the cable FH link. This wireless-wired resource mapping, referred to as Space-Frequency to SpaceFrequency (SF2SF) multiplexing due to the space-frequency nature of both wireless and wired signals, is shown in the paper to substantially mitigate the impairments introduced by the cable $\mathrm{FH}$, once evaluated in terms of UE throughput. An example is shown in Fig. 2.4, in which the RRU receives the signal from the single-UE, maps it onto the cable by exploiting the SF2SF multiplexing principle, and then relays it
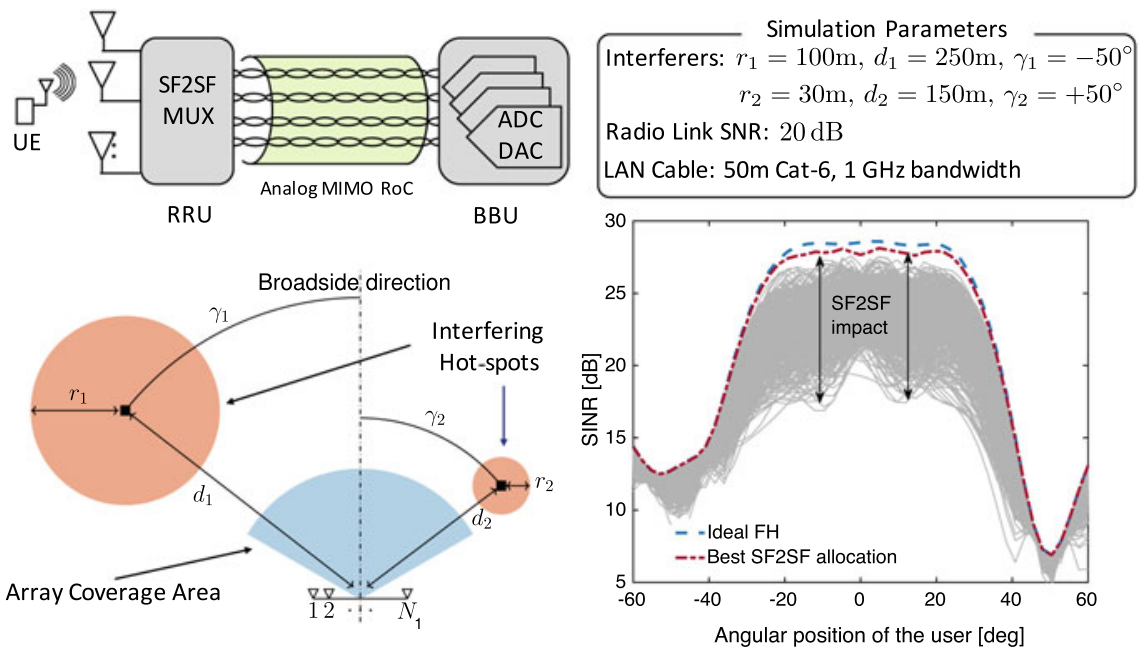

Fig. 2.4 Impact of SF2SF multiplexing on the single-user A-MIMO-RoC performance [14] (color figure online) 
to the BBU over the LAN cable. The SINR for the single-UE signal at the BBU is shown as performance metric by varying the angular position of the UE within the array coverage area, and assuming that two adjacent hotspots interfere with the useful signal. Each solid grey line in the plot is obtained by randomly selecting a SF2SF allocation at the RRU. For the purposes of this article, beside the expected performance losses in correspondence of the two interfering hotspots, it is enough to notice the great gain the can be achieved by selecting the best SF2SF at each angle (dashed-dotted red line) which attains the performance of the ideal mobile FH (dashed blue line). More details can be found in [14].

A similar scenario is considered in [15], where the coexistence between FH signals and other services (e.g., DSL, PoE, etc.) over the same LAN cable has been tested for indoor, thus confirming the performance boost provided by SF2SF multiplexing technique. Still focusing on the single-UE A-MIMO-RoC channel, [16] presents an information theoretical study for heterogenous $5 \mathrm{G}$ networks, whereby enhanced Mobile Broadband (eMBB) and Ultra Reliable Low Latency Communications (URLLC) services coexist in the same physical resources. The analysis in [16] provides some useful insights on the performance trade-offs between the two services when they coexist in the uplink of the proposed A-MIMO-RoC architecture.

The problem of optimally designing SF2SF multiplexing is tackled for the AMIMO-RoC downlink channel in single- and multi-UE settings in [17] and [18], respectively. In addition to all the issues due to the analog relaying of signals already mentioned for the uplink channel, the downlink SF2SF problem is complicated by the different power constraints that need to be jointly fulfilled both at the cable input and at the RRU antennas. Furthermore, in multi-UE settings digital precoding at the BBU needs to be properly designed in order to cope with the resulting multi-UE interference. As a first step, [17] confirms the potential of SF2SF also for the singleUE downlink A-MIMO-RoC channel. Then, [18] shows that, in multi-UE settings, the SF2SF multiplexing optimization (performed at the RRU), jointly designed with digital precoding of the overall wired-plus-wireless channels (performed at the BBU) and UE ordering optimization, is able to cope with both multi-UE interference and analog FH impairments, thus providing substantial performance gains in terms of minimum rate guaranteed for all the UEs. Finally, [19] focuses on the precoder design problem proposing a nearly-optimal BBU precoding algorithm and RRU power amplification strategy for the multi-UE A-MIMO-RoC downlink channel.

\subsection{A-MIMO-RoC Prototype and Experimental Results}

The A-MIMO-RoC prototype platform has been purposely developed in order to validate by real-world experiments the proposed FH architecture. The goal of this section is to describe part of the experiments that have been conducted in order to: $(i)$ confirm the transport capabilities offered by the pre-existing copper cabling infrastructure of buildings, and (ii) experimentally prove the potential of the proposed SF2SF wireless-wired resource allocation strategy, expected to enable A-MIMO- 
RoC to transparently carry multi-antenna RF signals over a single LAN cable. In particular, this is achieved by demonstrating the possibility to relay MIMO LTE signals, in an all-analog fashion, over LAN cables exploiting also high cable-frequency (tested here up to $400 \mathrm{MHz}$ ) with negligible performance degradation.

\subsubsection{Experimental Settings}

The A-MIMO-RoC prototype, shown in Fig. 2.5, is composed by two identical bidirection LAN-to-Coax Converters (L2CCs). The prototype platform has been tested through the TRIANGLE testbed, the essence of the H2020 TRIANGLE project [20], that allowed us to experimentally measure the end-to-end performance degradation introduced by the analog relaying over the copper-cable that is interposed between the two L2CCs. The LTE signals to be relayed over copper have been generated by the Keysight UXM RAN emulator.

The TRIANGLE testbed has been employed in its typical device-testing configuration, but inserting a 4-pairs RJ45 LAN cable between the RF output ports of the UXM and the RF connections at the UEs. The cable interfaces with the UXM and the UEs by means of the two L2CCs, which represent the core of the A-MIMO-RoC platform. The L2CCs perform impedance adaptation, cable equalization, coax-to-pairs mapping/demapping and RF/IF conversion (i.e., that implement SF2SF functionalities), and include all-passive/all-analog devices along the signal path to ensure fully bi-directional operations.

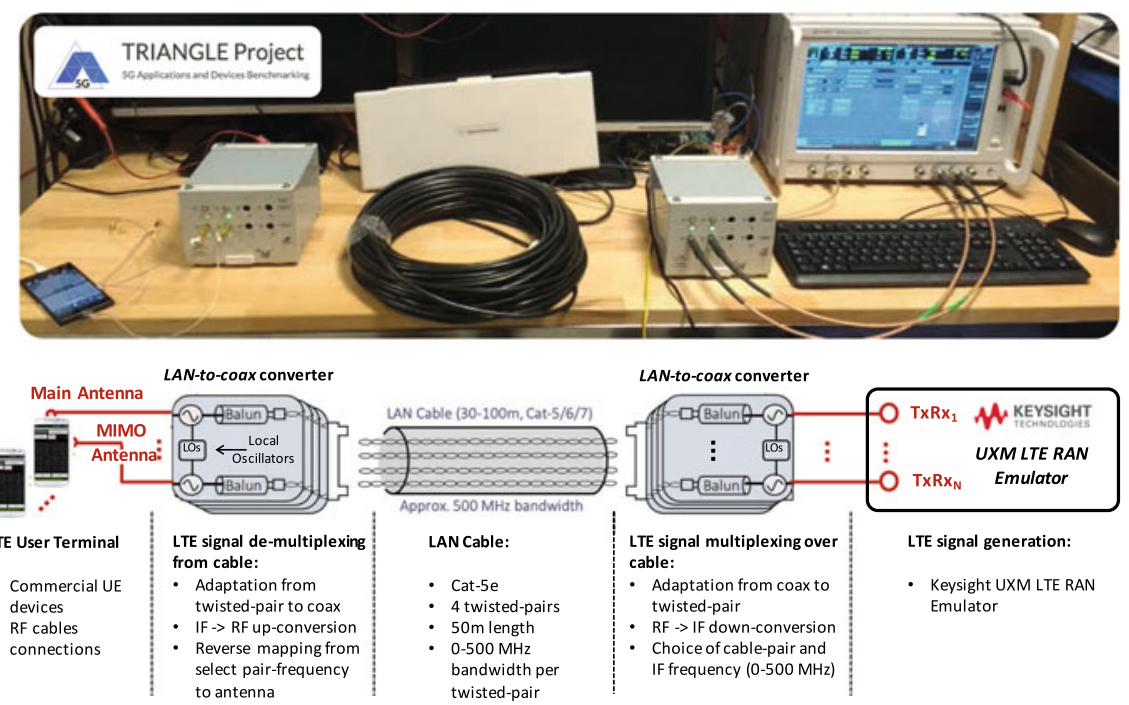

Fig. 2.5 A-MIMO-RoC prototype and experiment setup 
For all wireless communications performed by exploiting the A-MIMO-RoC platform, we used a $50 \mathrm{~m}$ Cat-5e LAN cable (which is the cable type commonly deployed in buildings) with a bandwidth experimented here up to $400 \mathrm{MHz}$ per each twistedpair. For the LTE signals, RF cables have been used to connect the second L2CC (i.e., the one on the left of Fig. 2.5) to the UE.

The top of Fig. 2.5 shows a simplified block diagram of the experimental setup, the bottom details the role of each component used for the experiments. In particular, the experiment setup is as follows (only the downlink is described, uplink is symmetrical): ( $i$ ) up to 4 LTE signals are generated by the UXM; (ii) RF cables are connected at each RF output of the UXM; (iii) the signal carried on each RF cable is IF-converted to match the LAN cable bandwidth (e.g., in the 10-400 MHz range), possibly multiplexed in frequency over cable by the first L2CC; (iv) each IF-converted signal is conveyed by one of the 4 twisted-pairs: cable adaptation/equalization, coax-to-pairs mapping and RF-to-IF conversion between coax and twisted-pair are performed by the first $\mathrm{L} 2 \mathrm{CC} ;(v)$ at the other end of the cable, RF cables are connected to the RF connectors of the second L2CC, that performs cable adaptation/equalization, pairsto-coax de-mapping and IF-to-RF conversion to interface with the UEs under test; (vi) the DEKRA Performance Tool and the TestelDroid test suite, integrated into the TRIANGLE testbed, are used to test the UEs performances [20].

\subsubsection{A-MIMO-RoC for LTE Versus Cable IF}

This experiment concerns the tests about relaying over copper a $2 \times 2$ MIMO LTE signal. The goal is two-fold: (i) to prove the feasibility of transporting 2 LTE RF bands, corresponding to the MIMO signal, over 2 different twisted-pairs of the LAN cable, but at the same cable IF $\left(f_{\mathrm{IF}}\right)$ to validate the extreme case of a strong mutual interference between two twisted-pairs (see Fig. 2.6); and (ii) to evaluate the performance degradation caused by increasing $f_{\mathrm{IF}}$, i.e., by using $f_{\mathrm{IF}}$ for which interference among pairs and attenuation are more severe.

Performance have been evaluated in downlink direction in terms of throughput and BLER for different MCSs, from 0-QPSK to 17-16QAM [22]. Figure 2.7 shows

\begin{tabular}{|l|l|}
\hline \multicolumn{2}{|c|}{ LTE System Settings } \\
\hline Signal Generation & Keysight UXM RAN Emulator \\
\hline LTE Band & B38 TDD, 2570-2620 GHz \\
\hline Bandwidth $\left(B W_{L T E}\right)$ & $5 \mathrm{MHz}$ \\
\hline Antenna Config. & $2 \times 2 \mathrm{MIMO}$ \\
\hline Transmission Mode & TM3 \\
\hline Radio Channel & ETSI Static MIMO \\
\hline TX Power & $-20 \mathrm{dBm}$ \\
\hline
\end{tabular}

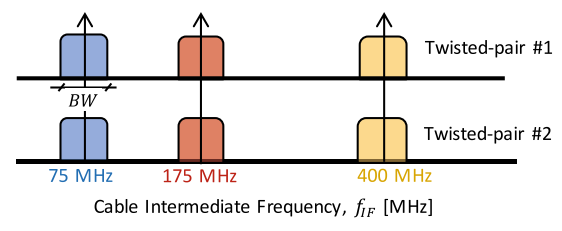

Fig. 2.6 A-MIMO-RoC experiment settings and RF signals mapping over cable 

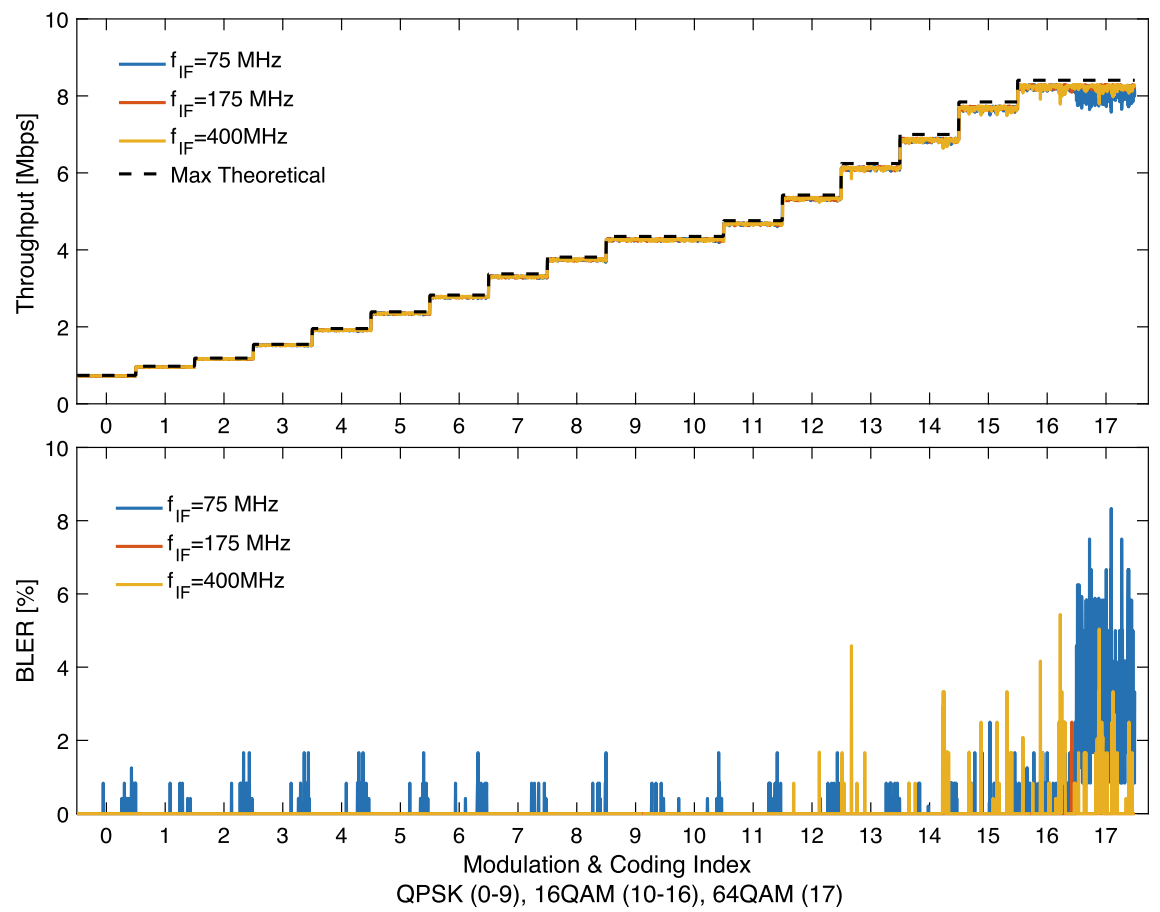

Fig. 2.7 Experiments for a $2 \times 2$ MIMO LTE signal: BLER and throughput versus MSC and IFs [21]

both throughput and BLER for a Static MIMO channel, $B W_{L T E}=5 \mathrm{MHz}$ and three different cable $f_{\mathrm{IF}}=75,175,400 \mathrm{MHz}$. For brevity, this is the only configuration shown in this article. However, additional results for LTE signals with different MIMO channel models and RF bandwidths are in [21]. The LTE system settings and signal mapping over cable adopted for the tests are reported in Fig. 2.6. The maximum theoretical throughput achievable by each MCS [22] over the considered channel bandwidth BW is shown as reference. Figure 2.7 proves that the performance loss due to the A-MIMO-RoC prototype is almost negligible for all considered MCS and cable $f_{\mathrm{IF}}$ selections. As expected, BLER increases for high MCS, but the degradation w.r.t the maximum throughput is still small. Concluding, Fig. 2.7 confirms experimentally the feasibility of relaying MIMO LTE signals over copper-cables at high frequency, even in the worst case of 2 LTE bands carried over 2 twisted-pairs at the same $f_{\mathrm{IF}}=400 \mathrm{MHz}$, and thus maximally interfering with each other. 


\subsection{Conclusions}

This article summarized the content of my Ph.D. thesis by presenting the Analog MIMO Radio-over-Copper (A-MIMO-RoC) architecture: an analog Cloud Radio Access Network (C-RAN) architecture that exploits analog FrontHaul (FH) links based on pre-existing copper-cables, e.g., Local Area Network (LAN) cables, to distribute Radio-Frequency (RF) signals into buildings at low cost, complexity and latency. The article first introduced the A-MIMO-RoC architecture by showing simulation results that assert the feasibility of LAN cables to efficiently relay RF indoors over the last $50-200 \mathrm{~m}$. Then, several previous works have been briefly reviewed in order to present the theory behind A-MIMO-RoC. These works cover multiple aspects of A-MIMO-RoC including wired-wireless resource allocation strategies for uplink and downlink channels, interference mitigation techniques for single- and multi-user settings, and the investigation of performance trade-offs for heterogeneous $5 \mathrm{G}$ networks with multiple services coexisting in the same physical resources. The overall theoretical discussion has been finally supported by experimental results obtained with a hardware prototype platform. These confirmed that A-MIMO-RoC is not only an interesting research topic providing numerous theoretical insights, but mainly a practical solution capable to cope with real-world problems that engineers and researchers are facing today in deploying next generation $5 \mathrm{G}$ indoor networks.

Acknowledgements Part of this work has been funded by the TRIANGLE project, European Union Horizon 2020 research and innovation programme, grant agreement No 688712. The part of this work about 5G RAN functional split options is the result of a fruitful interaction with Nokia Bell Labs and their support is acknowledged. Last but not least, many thanks go to my Ph.D. supervisor Prof. Umberto Spagnolini for his continuous support and guidance.

\section{References}

1. Matera A (2019) Interference mitigation techniques in hybrid wired-wireless communications systems for cloud radio access networks with analog fronthauling

2. CommScope (2016) Wireless in buildings: what building professionals think, Report

3. Checko A et al (2015) Cloud RAN for mobile networks: a technology overview. Commun Surv Tuts 17(1):405-426

4. CPRI Specifications V.6.1 (2014-07-01), September 2014

5. 3GPP TSG RAN (2017) TR 38.801 v14.0.0, Study on new radio access technology: radio access architecture and interfaces (Release 14)

6. Wake D et al (2010) Radio over fiber link design for next generation wireless systems. J Lightw Technol 28(16):2456-2464

7. Gambini J et al (2013) Wireless over cable for femtocell systems. IEEE Commun Mag 51(5):178-185

8. Lu C et al (2014) Connecting the dots: small cells shape up for high-performance indoor radio. Ericsson Rev 91:38-45

9. Weldon MK (2016) The future X network: a Bell Labs perspective. CRC Press, Boca Raton

10. HKT, GSA, Huawei, "Indoor 5G Networks - White Paper," Sept. 2018

11. Naqvi SHR et al (2017) On the transport capability of LAN cables in all-analog MIMO-RoC fronthaul. In: IEEE WCNC. IEEE, pp 1-6 
12. Huang Y et al (2015) LTE over copper-potential and limitations. In: IEEE PIMRC. IEEE, pp 1339-1343

13. Medeiros E et al (2016) Crosstalk mitigation for LTE-over-copper in downlink direction. IEEE Commun Lett 20(7):1425-1428

14. Matera A et al (2017) Space-frequency to space-frequency for MIMO radio over copper. In: IEEE ICC. IEEE, pp 1-6

15. Matera A et al (2017) On the optimal space-frequency to frequency mapping in indoor singlepair RoC fronthaul. In: EuCNC. IEEE, pp 1-5

16. Matera A et al (2018) Non-orthogonal eMBB-URLLC radio access for cloud radio access networks with analog fronthauling. Entropy 20(9):661

17. Matera A et al (2018) Analog MIMO-RoC downlink with SF2SF. IEEE Wirel Commun Lett $1-1$

18. Matera A et al (2019) Analog MIMO radio-over-copper downlink with space-frequency to space-frequency multiplexing for multi-user $5 \mathrm{G}$ indoor deployments. IEEE Trans Wirel Commun 18(5):2813-2827

19. Rizzello V et al (2019) Precoding design for the MIMO-RoC downlink. In: IEEE ICASSP 2019, pp 4694-4698

20. Cattoni AF et al (2016) An end-to-end testing ecosystem for 5G. In: EuCNC. IEEE, pp 307-312

21. Matera A et al (2019) Analog MIMO radio-over-copper: prototype and preliminary experimental results. In: ISWCS 2019. IEEE

22. 3GPP TS 36.213 group radio access network; Evolved universal terrestrial radio access (EUTRA), "Physical layer procedure."

Open Access This chapter is licensed under the terms of the Creative Commons Attribution 4.0 International License (http://creativecommons.org/licenses/by/4.0/), which permits use, sharing, adaptation, distribution and reproduction in any medium or format, as long as you give appropriate credit to the original author(s) and the source, provide a link to the Creative Commons license and indicate if changes were made.

The images or other third party material in this chapter are included in the chapter's Creative Commons license, unless indicated otherwise in a credit line to the material. If material is not included in the chapter's Creative Commons license and your intended use is not permitted by statutory regulation or exceeds the permitted use, you will need to obtain permission directly from the copyright holder.

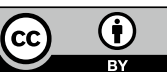

